\title{
Compactness Measurement Using Fuzzy Multicriteria Decision Making For Redistricting
}

\author{
Wang Yin Chai, Bong Chin Wei
}

\begin{abstract}
This paper presents a new method for compactness assessment in redistricting planning using Fuzzy Multicriteria Decision Making. An Enhanced Compactness Index (ECI) representing the overall plan with respect to each criterion is obtained by using triangular fuzzy number. The ECI is generated based on the synthesis of the concepts of fuzzy set theory, AHP, $\alpha$-cuts concept and index of optimism of district planners to estimate the degree of satisfaction of the judgements on a district plan. The proposed method is more flexible, simple and comprehensive with easy computation and efficiency which facilitates its uses in compactness measurement in redistricting application like school redistricting, election boundary redistricting and others. A case study on forest blocking is presented to demonstrate its applicability in redistricting applications with respect to their redistricting goals and criteria.
\end{abstract}

Index Terms: Artificial Intelligence, Fuzzy System, and Geographical Information System

\section{INIRODUCTION}

$\mathrm{T}$ he aim for redistricting or districting is to foster community and it begs the tough question: where to draw the lines for the district? It is tremendous important that it reacts on how to take control on the space of a particular region. Nonetheless, current redistricting method tends to produce odd and bizarre shape of districts, which often characterised as noncompact district plan. Compactness measurement is useful to measure the compactness and continuity of a district plan. However, choosing the compactness measurements is a special challenge because there are over thirty distinct measures of compactness. Thus, this paper aims to enhance existing compactness measurements and use it in redistricting algorithm to improve the shape of the district plan.

Subsequently, this paper discusses on the use of Fuzzy Multicriteria Decision Making (FMCDM) on compactness measurement in redistricting process. In simple, the aims of this paper is as the following:
1) To produce better shape assessment index according to integration of multiple compactness measurement method that is more descriptive and able to incorporate with natural feelings of dis trict planners with fuzziness.

2) To incorporate the new index into redistricting to generate an optimal compact district.

3) To consider the restricted boundary like river, political boundary, and others during redistricting.

4) To work and perform in an environment that is able to manage the spatial and non-spatial data, their relationship and dependency.

Fig 1 briefly shows the revised enhanced redistricting model in terms of the input, process and output. Thus, the input data are both the spatial data and the related non-spatial data. The domain of the proposed model is on enhancing the decision support system in maintaining the compactness and continuity of redistricting to produce the optimal compact district plan with FMCDM. Meanwhile, GIS technology acts as a supportive tool because of its capability to handle the relationships of spatial data between non-spatial or namely attributes data.

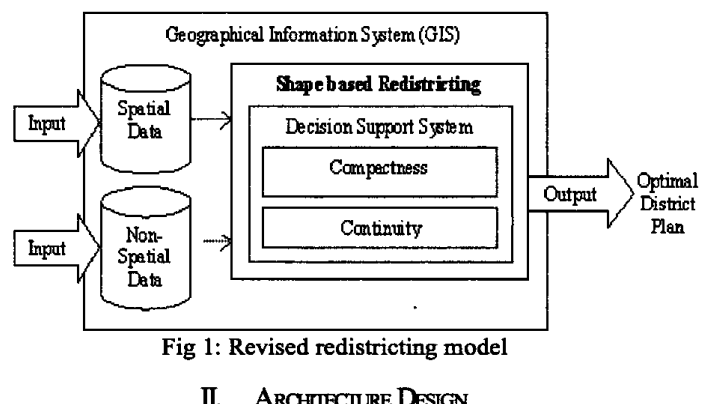

This paper applies the concept of FMCDM at each redistricting stage shown in the semantic diagram in Fig 2. Shape Optimal Rules (SOR) is the data store, which consists of the knowledge acquisition from the district planners. This data store includes the objective and the fuzzy rules sets for the FMCDM. Two other data stores: Application Dependent Data Store (ADD) and Application Independent Data Store (AID) provide the necessary input data for the algorithm. The initiate stage is in Data Preparation Module (DPM), followed by three 